\title{
LOW AND HIGH ENTHALPY SHOCK WAVE / BOUNDARY LAYER INTERACTIONS AROUND CYLINDER-FLARE MODELS
}

\section{B. Chanetz}

Fundamental and Experimental Aerodynamics Department ONERA, Meudon 92190, France

The paper describes four test-cases around the same configuration type: a hollow cylinder flare model. The first three test-cases are relative to cold hypersonic flow and the fourth one to hot hypersonic flow: $(i)$ a purely laminar low enthalpy interaction performed at Mach 9.92; (ii) a transitional low enthalpy interaction performed at Mach 5; (iii) a fully low enthalpy turbulent interaction performed at Mach 5; and $(i \nu)$ a high enthalpy interaction performed at Mach 9.4.

\section{INTRODUCTION}

In spite of the spectacular progress in computational fluid dynamics (CFD) there is still a strong need to validate computer with experiments. Because of computational cost, it is better to consider two-dimensional (2D) configurations. A truly $2 \mathrm{D}$ configuration is difficult to realize in a wind tunnel due to starting constraints which forbid the use of large spanwise models. These experiments have been executed on axisymmetric configurations. The experiments presented here are from the fundamental and experimental aerodynamics department at ONERA Meudon [1]. They are invaluable to the development of reliable and accurate codes.

This paper provides four test-cases around the same model shape: a hollow cylinder flare model. In such a model are avoided the side effects due to the finite span of any $2 \mathrm{D}$ arrangement but the mathematical simplicity of a $2 \mathrm{D}$ space is maintained. An axisymmetrical configuration facilitates a rigorous numerical study with the current computational capabilities. These models relative to academic configurations have been chosen in order to simulate the shock wave/boundary layer interactions occurring on flaps. Indeed, shockwave/boundary-layer interactions in hypersonic flows may have major consequences on thermal loads, especially if the shock is strong enough to induce

This is an Open Access article distributed under the terms of the Creative Commons Attribution-Noncommercial License 3.0, which permits unrestricted use, distribution, and reproduction in any noncommercial medium, provided the original work is properly cited. 
separation. Furthermore, the heat-flux density levels in the interaction region strongly depend on the nature (laminar, transitional, or turbulent) of the boundary layer. The phenomena are still more difficult when reacting gas hypersonic effects are. Therefore, this paper presents laminar, transitional, and turbulent shock-wave boundary layer interactions in cold hypersonic flow as well as interactions in hot hypersonic flow.

\section{LOW ENTHALPY LAMINAR INTERACTION}

\subsection{Goal of the Study and Experimental Conditions}

Entirely laminar flows have been realized in the low Reynolds number R5Ch wind tunnel whose operating conditions are: Mach number $\mathrm{M}_{0}=9.92$, stagnation temperature $T_{\text {st }}=1050 \mathrm{~K}$, and stagnation pressure $p_{\mathrm{st}}=2.5 \cdot 10^{5} \mathrm{~Pa}$. The unit Reynolds number $\left(186,000 \mathrm{~m}^{-1}\right)$ is sufficiently low to warrant a laminar regime throughout the interaction domain.

The model consists of a hollow cylinder, with a sharp leading edge, followed by a flare cylinder. The model has a total length of $0.17 \mathrm{~m}$, the reference length, based on the distance between the sharp leading edge and the beginning of the 30 degree flare, is equal to $L=0.1017 \mathrm{~m}$. The outer cylinder diameter is equal to $0.065 \mathrm{~m}$ and the inner cylinder diameter is equal to $0.045 \mathrm{~m}$. The Reynolds number based on the reference length is equal to $\operatorname{Re}_{L}=18,600$.

The flow has been visualized using Electron Beam Fluorescence (EBF) [2]. The wall pressure measurements were executed with variable reluctance differential transducers and the heat fluxes determined from the surface temperature rise using platinum films. In addition to wall measurements, density and specific mass measurements were performed using electron beam X-ray techniques [2].

The Knudsen number $\mathrm{Kn}=\lambda / L$ (with $\lambda$ the mean free path and $L$ the reference length) in equal to 0.001 . This is a sufficiently low value to assume the validity of continuum regime and the approach with Navier-Stokes equations. This is also a convenient value to use the Direct Molecular Simulation well adapted to high Knudsen number.

So, the present results are compared with calculated data issued from three different solvers [3]:

(1) a Navier-Stokes research solver from ONERA: NASCA (finite volumes);

(2) a Navier-Stokes code from DLR: FLOW (finite elements); and

(3) a Direct Simulation Monte-Carlo solver from NASA Langley: DSMC. 


\subsection{Results}

Figure 1 shows an EBF visualization with an attached shock-wave at the sharp leading edge and a separation shock wave. These two shocks converge and interfere above the end of the flare. Three density profiles were probed at $X / L=0.3$, 0.6 , and 0.76 .

Wall pressure measurements are transformed into a pressure coefficient:

$$
C_{p}=\frac{2\left(p / p_{0}-1\right)}{\gamma \mathrm{M}_{0}^{2}}
$$

where $\mathrm{M}_{0}$ and $p_{0}$ are the upstream Mach number and upstream static pressure.

Figure $2 a$ presents the longitudinal evolution of the pressure coefficient measured on the hollow cylinder-flare model for ten runs. The pressure coefficient decreases slowly on the upstream part of the cylinder until the abscissa $X / L=0.7$. This decrease is followed by a two-step compression. A first compression is due to the separation process and a second compression, far more important, occurs during reattachment. The Navier-Stokes codes give a fair prediction of the wall pressure distribution in the separated flow, whereas the DSMC code highly overpredicts the peak pressure.

Wall heat-flux densities $q_{w}$ are transformed into a thermal coefficient

$$
h=\frac{q_{w}}{T_{\mathrm{st}}-T_{w}}\left[\mathrm{~W} / \mathrm{m}^{2} / \mathrm{K}\right]
$$

where $T_{\mathrm{st}}$ is the stagnation temperature and $T_{w}$ is the wall temperature at the beginning of the run. The results are expressed in a dimensionless form by introducing the Stanton number defined by:

$$
\mathrm{St}=\frac{h}{\rho_{0} U_{0} C_{p}}
$$

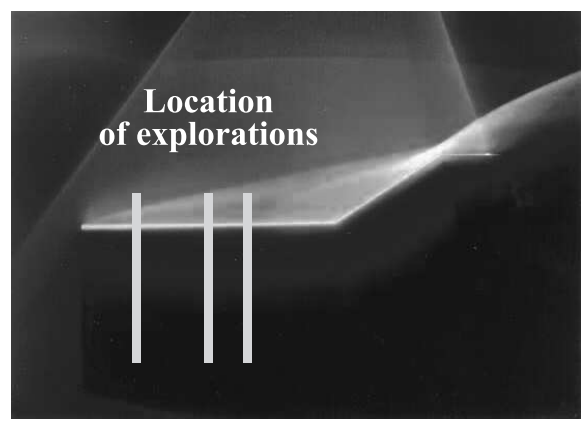

Figure 1 Electron beam fluorescence visualization (low enthalpy laminar interaction) 


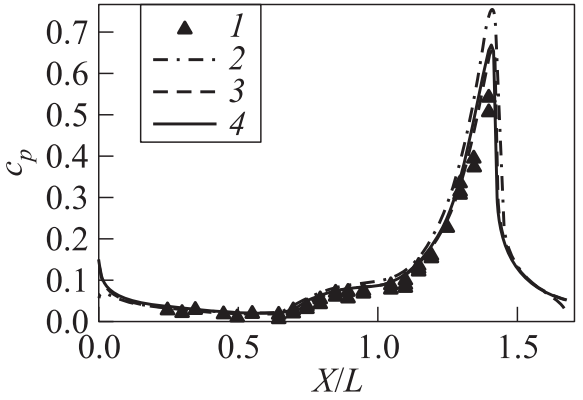

(a)

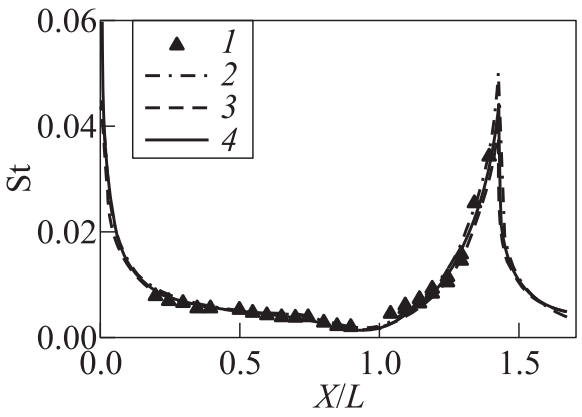

(b)

Figure 2 Surface measurements: $(a)$ pressure; and (b) heat-transfer (low enthalpy laminar interaction) $(1$ - experiments; 2 - DSMC; 3 - FLOW; and 4 - NASCA $)$

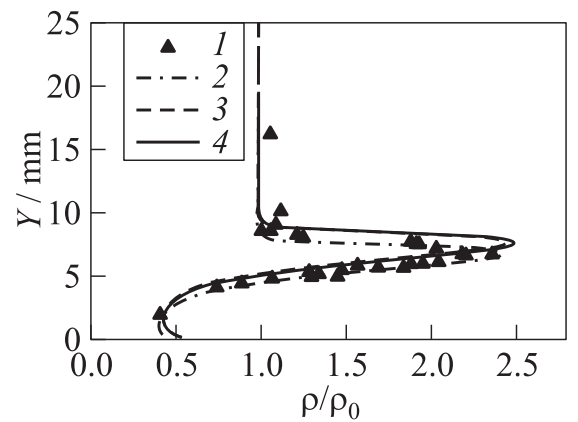

(a)

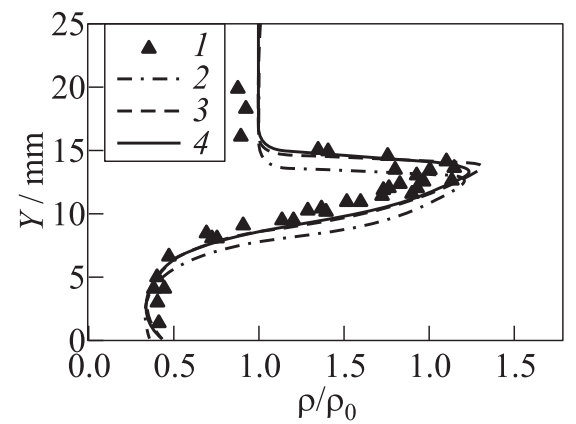

(b)

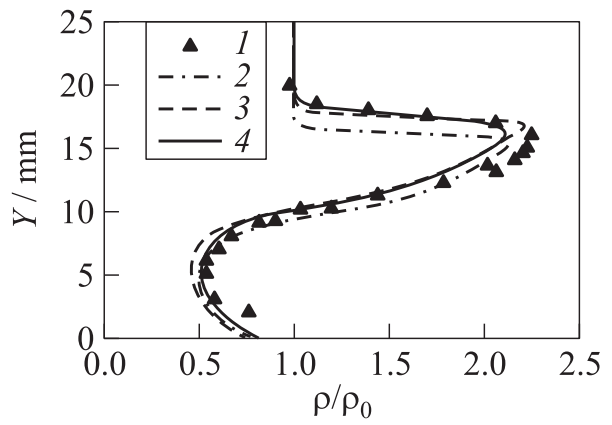

(c)

Figure 3 Density profile measurements (low enthalpy laminar interaction) (1 experiments; 2 - DSMC; 3 - FLOW; and 4 - NASCA): $(a) X / L=0.3 ;(b) 0.6$; and $(c) X / L=0.76$ 
where $C_{p}=1004.5 \mathrm{~J} / \mathrm{kg} / \mathrm{K}$ and $\rho_{0}$ and $U_{0}$ are inferred from the upstream conditions.

Figure $2 b$ presents the evolution of the Stanton number along the cylinder flare. The heat flux decreases along the upstream part of the cylinder where the flow is governed by the viscous interaction effects emanating from the leading edge. Further downstream, the evolution is characteristic of a laminar interaction with a large boundary layer separation: after an initial decrease in the heat-flux evolution beginning at the abscissa where the separation begins $(X / L=0.77)$, this flux increases on the flare, with an enforcement of this increase from the reattachment point.

Besides wall measurements, field quantities have been measured around the model. As shown in Fig. 3, the experimental density profiles are in good agreement with the calculations. For these stations, located forward of the separation line, the increase in density is due to the shock generated by the sharp leading edge.

\section{LOW ENTHALPY TRANSITIONAL INTERACTION}

\subsection{Experiment Conditions}

A hollow cylinder-flare model was used. It is composed of a cylinder with sharp leading edge (diameter $D=0.131 \mathrm{~mm}$ and length $L=0.252 \mathrm{~m}$ ) followed by a 15 degree conical flare (length $101.4 \mathrm{~mm}$ ). The flare itself is followed by a 0.05meter long cylindrical extension in order to minimize the base flow influence on the interaction region. Total length of the model is $349 \mathrm{~mm}$. The ONERA Meudon center R2Ch wind tunnel was used for these experiments. R2Ch wind tunnel is a blowdown facility (test duration between 15 and $30 \mathrm{~s}$ ) equipped with a Mach 5 contoured axisymmetric nozzle. Upstream air is heated to a stagnation temperature $T_{\text {st }}$ of approximately $500 \mathrm{~K}$ by streaming through a Joule effect heater. Stagnation pressure $p_{\text {st }}$ can be adjusted between $0.9 \cdot 10^{5}$ and $70 \cdot 10^{5} \mathrm{~Pa}$. $\mathrm{R} 2 \mathrm{Ch}$ wind tunnel is — as $\mathrm{R} 5 \mathrm{Ch}$ - a cold hypersonic facility, in the sense that the stagnation temperature is raised to a level just sufficient to prevent air liquefaction during expansion in the nozzle. These tests were performed for a stagnation pressure $p_{\mathrm{st}}=0.9 \cdot 10^{5} \mathrm{~Pa}$, which corresponds to a Reynolds number $\operatorname{Re}_{L}=0.38 \cdot 10^{6}$ based on the reference length $L$.

For axisymmetric configurations, the separation line is very sensitive to the model incidence and yaw angles. Great care was taken to control model setting in the flow by examining the surface flow with the help of viscous coating visualizations. This technique uses a mixture of black lamp and silicone oil, with a viscosity suited to the local skin friction level painted, onto the model before the test. The skin friction line patterns established during the run allows for 


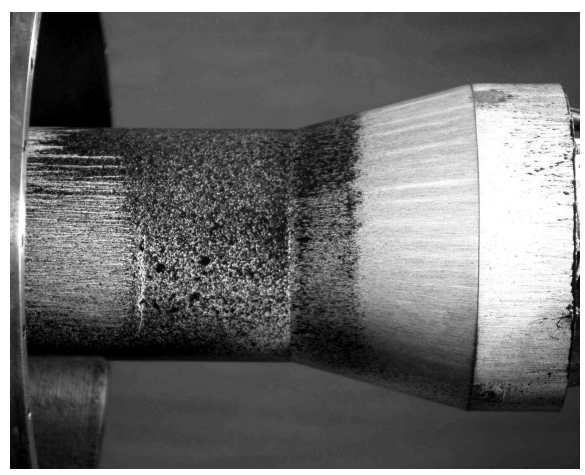

Figure 4 Surface flow visualization (low enthalpy transitional interaction)

identification of separation and reattachment lines, thus permitting a check the flows axial symmetry (Fig. 4).

Pressure and heat-fluxes have been measured by pressure transducers and heat-flux sensors. The velocity field has been probed by two-component Laser Doppler Velocimetry (LDV). The boundary layer was probed at different stations upstream, inside, and downstream of the interaction region. In the present case, the probe volume is a circular ellipsoid having a major axis of few millimeters and a minor axis of $0.280 \mathrm{~mm}$. The minimum probing distance to the wall was approximately $0.4 \mathrm{~mm}$. The uncertainty for LDV measurements inside the boundary layer is about $1 \%$ of the maximum velocity modulus of the upstream external flow $U_{0}(830 \mathrm{~m} / \mathrm{s})$. In the external, high-velocity flow, due to the particle drag, this uncertainty icreases up to $5 \%$ of $U_{0}$.

\subsection{Results}

The surface flow visualization represented in Fig. 4 shows the separation (at $X / L=0.73$ ) and reattachment lines (at $X / L=1.23$ ).

The location of separation agrees with computational predictions by a laminar calculation performed with the ONERA-NASCA solver.

Wall pressure and heat-flux distributions measured in the $\mathrm{R} 2 \mathrm{Ch}$ wind tunnel are compared (Figs. 5 and 6) with the calculated values obtained with the NASCA code for laminar conditions. Concerning the separation point, good agreement is obtained between experiment and calculation. The pressure level in the reverse flow zone is also well predicted by the calculation. As far as the heat-flux distribution is concerned, a rough agreement between calculation and experiment is observed until $X / L=1$. However, the experimental heat-flux peak following the reattachment is about 2.5 times higher than the laminar cal- 


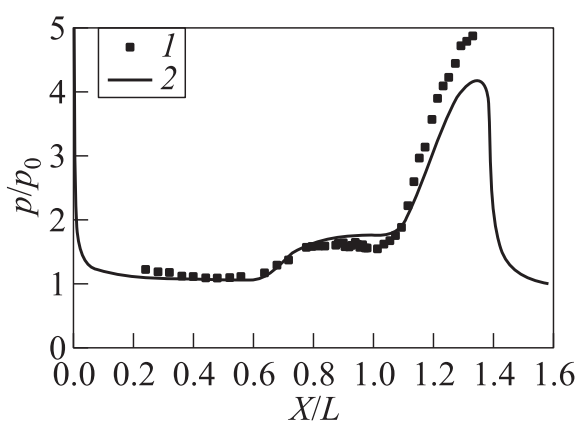

Figure 5 Wall pressure distribution (low enthalpy transitional interaction): 1 - experiments; and 2 - laminar calculation

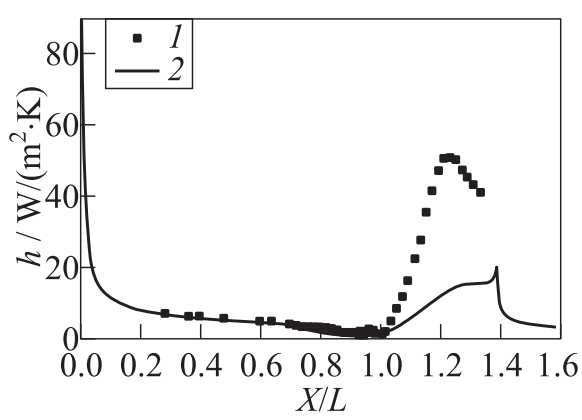

Figure 6 Wall heat-transfer distribution (low enthalpy transitional interaction): 1 - experiments; and 2-laminar calculation

culation. This result is in agreement with the well-known tendencies relative to the transitional thermal loads, which are larger than the laminar ones and even larger than the turbulent ones. These have been proven by experiments performed at Onera [4] for an identical Reynolds number in natural transition or with triggered transition.

The semilogarithmic plotting of the measured heat-flux density evolution in Fig. 7 emphasizes the important decrease of heat-flux density in the separation region typical of laminar separation. The laminar and turbulent wall heat fluxes calculated on a flat plate for the same stagnation conditions are induced in the

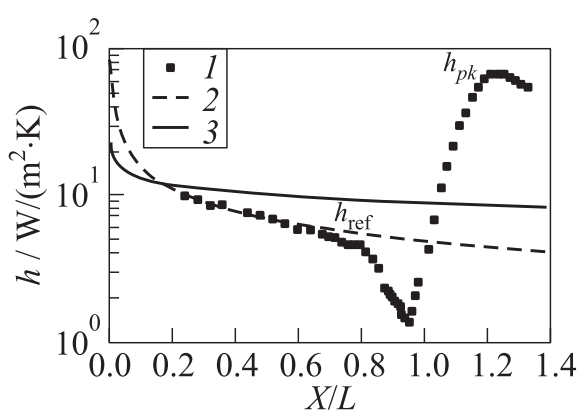

Figure 7 Wall heat-flux density distribution in semilogarithm plot (low enthalpy transitional interaction): 1 - experiments; 2 - laminar theory; and 3 - turbulent theory

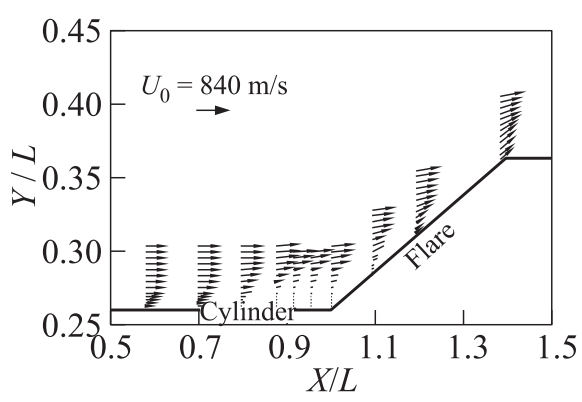

Figure 8 The LDV measurements (low enthalpy transitional interaction) 
figure. These calculations use the boundary layer theory [5] which furnishes the evolution of the Stanton number on a flat plate without separation in both situations: laminar or turbulent regime. Close agreement is observed between experiments and the theoretical laminar evolution up to the point of separation, which confirms the laminar nature of separation.

Results of the LDV measurements are shown in Fig. 8. In this case, the location of the separation is at $X / L=0.69$ according to the oil-flow visualization shown in Fig. 4. The incompressible shape factor for the first boundary-layer velocity profile located at $X / L=0.57$ is equal to $H_{i}=2.57$. This value is characteristic of a laminar boundary layer. At $X / L=0.69$ just upstream of the separation abscissa, the incompressible shape factor is still equal to $H_{i}=2.59$ confirming the laminar characteristic of the flow upstream of separation.

\section{LOW ENTHALPY TURBULENT INTERACTION}

\subsection{Experimental Conditions}

This test-case [6] was performed in the $\mathrm{R} 2 \mathrm{Ch}$ wind tunnel already described (see subsection 3.1). The present experiment was performed for the following conditions at $\mathrm{M}_{0}=5$ : stagnation pressure $p_{\text {st }}=35 \cdot 10^{5} \mathrm{~Pa}$ and stagnation temperature $T_{\mathrm{st}}=500 \mathrm{~K}$. These free stream conditions correspond to a unit Reynolds number equal to $44.1 \cdot 10^{6} \mathrm{~m}^{-1}$. The model is a hollow cylinder with an external diameter of $0.131 \mathrm{~m}$ and an internal diameter of $0.106 \mathrm{~m}$. The leading edge is sharp with an interior angle of $10^{\circ}$. A 35 degree flare is mounted $0.25 \mathrm{~m}$ downstream of the cylinder. The total length of the model is equal to $0.03 \mathrm{~m}$. The Reynolds number based on the reference length $L=0.25 \mathrm{~m}$ is equal to $\operatorname{Re}_{L}=11 \cdot 10^{6}$ and was sufficiently high to insure a natural fully turbulent boundary layer well upstream of the cylinder-flare junction.

\subsection{Presentation of the Results}

The wall pressure distribution is given in Fig. 9. Pressure $p$ measured at the wall is divided by the free stream infinite pressure $p_{0}$. Along the cylinder part, $p$ is nearly equal to $p_{0}\left(p / p_{0}=1\right)$ until abscissa $X / L=0.9$ where it increases significantly, which is a sign of turbulent separation. This feature is confirmed by the heat-flux distribution presented in Fig. 10, where an increase of the thermal coefficient is observed between $X / L=0.4$ and $X / L=0.5$.

Thus, the end of transition took place at $X / L=0.5,0.125 \mathrm{~m}$ downstream of the cylinder edge in the middle of the cylinder. Then, a separation of the boundary layer occurs before the flare in the fully turbulent regime. Heat transfer 


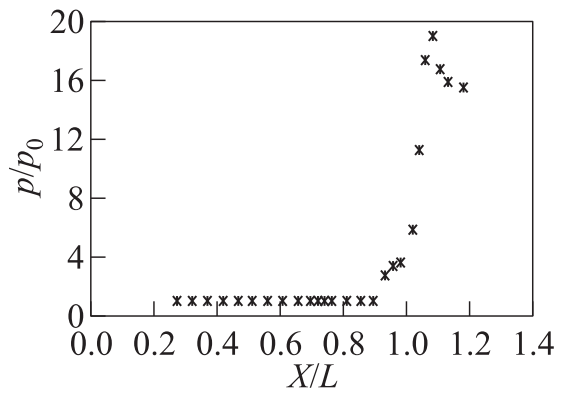

Figure 9 Wall pressure distribution (low enthalpy turbulent interaction)

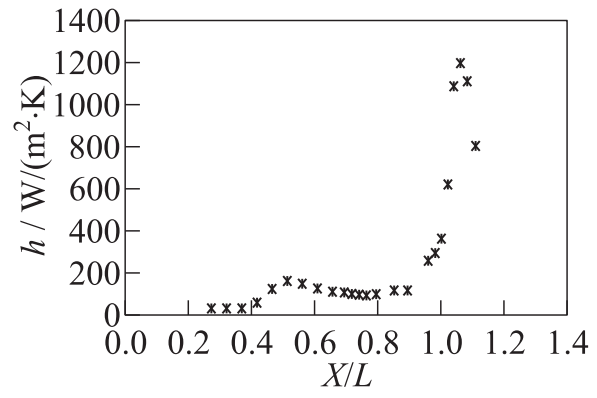

Figure 10 Wall heat-transfer distribution (low enthalpy turbulent interaction)

increases, which is typical of a separation onset in the turbulent regime. This experiment constitutes an interesting test-case for validation of computational turbulence models.

\section{HIGH ENTHALPY INTERACTION}

\subsection{Conditions of the Experiments}

This test case was also performed with a cylinder flare geometry composed of a hollow cylinder with a sharp leading edge, an external diameter of $0.15 \mathrm{~m}$, and a length $L=0.2 \mathrm{~m}$. The cylinder is followed by a 35 degree conical flare (length $0.067 \mathrm{~m}$ ). The flare itself is followed by a 0.033 -meter long cylindrical extension in order to minimize the base flow influence on the interaction region. To measure the pressure distribution, the model was equipped with DRUCK FDCR-42 transducers, 22 pressure taps were located along an upper generating line. To check the correct position of the model inside the chamber, the model was equipped with four additional control taps located symmetrically along the generating line at $\pm 90^{\circ}$ with respect to the original one. To measure the heat flux, the model was equipped with 22 thermocouples located along an upper generating line at $180^{\circ}$ from the upper generating line and at the same streamwise positions (of the pressure taps).

The Onera-F4 high-enthalpy wind tunnel was used for the present experiments. Two runs were performed at the same conditions. One in air and another with nitrogen in order to qualify an eventual effect of dissociation. In the conditions of the run with air, Onera-F4 high-enthalpy wind tunnel flow conditions at the nozzle exit are $\mathrm{M}_{\infty}=9.4, \mathrm{Re}_{\infty} / m=1.15 \cdot 10^{5}, T_{\infty}=522 \mathrm{~K}$, and $V_{\infty}=4318 \mathrm{~m} / \mathrm{s}$, thus corresponding to stagnation pressure $p_{\mathrm{st}}=230 \cdot 10^{5} \mathrm{~Pa}$ and total enthalpy $h_{\mathrm{st}}=9.93 \mathrm{MJ} / \mathrm{kg}$. 


\subsection{Presentation of the Results}

In order to compare the run performed in air with the run in nitrogen, it is necessary to consider the good appropriate parameters. The run duration is comprised between 200 and $300 \mathrm{~ms}$, but only the half of the duration is available for valuable measurements. During this duration, the stagnation conditions (pressure and enthalpy) are decreasing. In fact, for each run, it is possible to consider more than one hundred different conditions. The comparison between air and nitrogen is interesting to evaluate because of the effects due to the dissociation, since with air, the dissociation of $\mathrm{O}_{2}$ molecules occurs at $2500 \mathrm{~K}$, whereas with pure nitrogen the dissociation of $\mathrm{N}_{2}$ occurs at $4000 \mathrm{~K}$. In order to have a good comparison, it has been demonstrated [2] that one has to consider the same stagnation enthalpy and the same global viscous interaction parameter $\underline{X}=\mathrm{M} /\left(\operatorname{Re}_{L}\right)^{0.5}$. This parameter is inferred from the Pitot pressures measured at the nozzle exit. Thus, the following conditions have been chosen:

- for the run 848 with air: $p_{\mathrm{st}}=22,991,200 \mathrm{~Pa} ; h_{\mathrm{st}} /\left(r T_{a}\right)=125.7$; and $\underline{X}=0.0627 ;$ and

- for the run 849 with nitrogen: $p_{\mathrm{st}}=19,613,700 \mathrm{~Pa} ; h_{\mathrm{st}} /\left(r T_{a}\right)=125.3$; and $\underline{X}=0.0648$

where $h_{\mathrm{st}} /\left(r T_{a}\right)$ is the reduced enthalpy level in the quiet chamber $\left(h_{\mathrm{st}}\right.$ is the enthalpy corresponding to the stagnation conditions, $r$ is equal to 287 for the perfect gas, and $T_{a}$ is the atmospheric temperature). The longitudinal evolution of wall heat-flux density $\Phi$ expressed in $\mathrm{W} / \mathrm{m}^{2}$ is presented in Fig. $11 a$ and the longitudinal evolution of the pressure is presented in Fig. $11 b$ also in

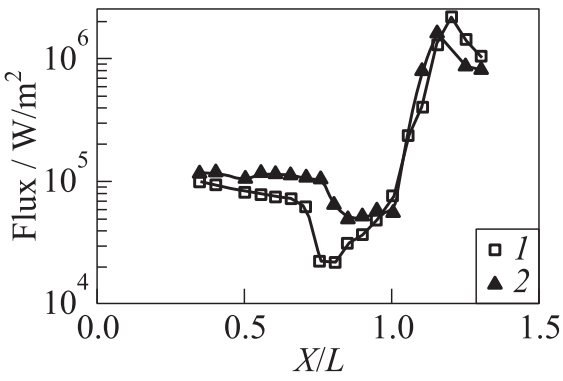

(a)

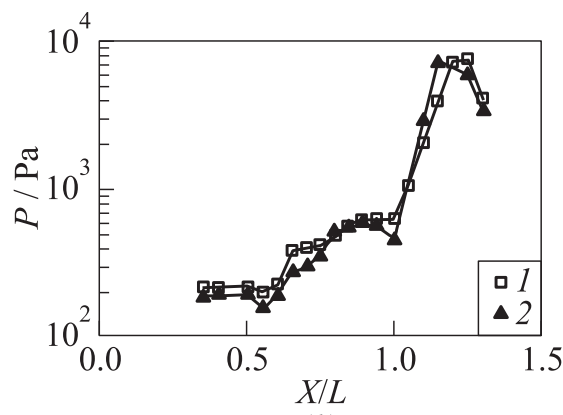

(b)

Figure 11 Wall heat-transfer $(a)$ and pressure $(b)$ distributions (high enthalpy interaction): $1-$ air, $P_{i}=229, H_{i}=126.7, \mathrm{M} / \mathrm{Re}^{0.5}=0.0527$; and 2 - nitrogen, $P_{i}=195$ 
semilogarithm plot. This presentation emphasizes the separation occurring before the ramp and characterized both by the slight decrease of heat flux and the correlative increase of pressure. With air - represented with squares - the separation point is found approximately at $X / L=0.7 \pm 0.01$ and with nitrogen - represented with triangles - the separation point is found approximately at $X / L=0.75 \pm 0.01$. The separation points are inferred from the curves. The difference is not very sensitive, but sufficient to demonstrate some dissociation effects. Indeed, the stagnation enthalpy is not sufficient to dissociate nitrogen molecules but sufficient to dissociate partly $\mathrm{O}_{2}$ molecules. Nevertheless, this study constitutes an interesting mean/high enthalpy test case for the validation of Navier-Stokes solvers in thermochemical desequilibrium [7].

\section{CONCLUDING REMARKS}

The first study presents an unquestionable shock wave / boundary layer test case in laminar flow in order to validate numerical solvers without considering the complex problem of turbulence modelling. The second test case presents an unusual transitional shock wave / boundary layer interaction test case. Indeed, the problem of transitional interaction was often avoided because its calculation was too difficult. But precisely such interactions give rise to larger heat-fluxes at reattachment, even higher than those obtained in fully turbulent regime. Therefore, this transitional well-documented test case [4] achieved at Onera fills a void in this domain. The third test case completes the range in cold hypersonic since it is relative to a natural fully turbulent shock wave / boundary layer interaction with transition occurring upstream of the separation. The fourth test-case is relative to high enthalpy hypersonic. The study performed in the F4 high-enthalpy wind tunnel has allowed a comparison between air and nitrogen for the similar flow conditions. This comparison may be useful to validate Navier-Stokes solvers in thermochemical inequilibrium.

However, a comparison restricted to the wall properties is generally insufficient to validate the most advanced predictive methods. In particular, information on the Mach number, temperature, and density fields is essential to elucidate the cause of discrepancies affecting the wall distribution. In these conditions, the validation of computer codes requires well documented experiments providing not only wall quantities but also flow field measurements. It is remarkable that the breakthrough in the predictive capacity has been paralleled by spectacular developments in measurement techniques over approximately the same period. Thus, two of the four test cases presented have been carefully investigated using the nonintrusive diagnostic techniques: LDV to measure velocity and X-ray EBF to measure density.

These four test cases constitute a powerful data base for hypersonic shock wave boundary layer interactions. 


\section{REFERENCES}

1. Benay, R., B. Chanetz, and J. Délery. 2003. Code verification: Validation with respect to experimental data banks. Aerospace Sci. Technol. 7:239-62.

2. Gorchakova, N., B. Chanetz, T. Pot, R. Bur, J.-P. Taran, D. Pigache, J. Moss, D. Schulte, L. Kuznetsov, and V. Yarygin. 2002. Progress in shock wave-boundary layer interaction studies in rarefied hypersonic flows using electron-beam-excited X-ray detection. AIAA J. 40(4):593-98.

3. Chanetz, B., R. Benay, J.-M. Bousquet, R. Bur, T. Pot, F. Grasso, and J. Moss. 1998. Experimental and numerical study of the laminar separation in hypersonic flow. Aerospace Sci. Technol. 3:205-18.

4. Benay, R., B. Chanetz, B. Mangin, L. Vandomme, and J. Perraud. 2006. Shock wave transitional boundary-layer interactions in hypersonic flow. AIAA J. 44(6).

5. Lengrand, J. C., and B. Chanetz. 2005. Boundary layer. Course in the Ecole Centrale de Paris.

6. Chanetz, B. 1996. ONERA hypersonic test-cases in the framework of Working Group 18. Hypersonic experimental and computational capability. AGARD Advisory Report 319. Vol. II (ONERA TP No. 1996-91).

7. Grasso, F., S. Cuttica, G. Ranuzzi, M. Marini, and B. Chanetz. 2001. Shock wave turbulent boundary layer interactions in non-equilibrium flows. AIAA J. 39(11). 